\title{
Intelligent Measurement and Monitoring of Carbon Emissions for 5G Shared Smart Logistics
}

\author{
Ailing Zhang $\mathbb{D}^{1},{ }^{1}$ Sha $\mathrm{Li}^{1}{ }^{1}$ Lin Tan, ${ }^{1}$ Yingchao Sun, ${ }^{2}$ and Fuxiao Yao ${ }^{1}$ \\ ${ }^{1}$ Shandong Vocational and Technical University of International Studies, Rizhao Shandong 276800, China \\ ${ }^{2}$ Qingdao Administrative Service and Public Resources Trading Center, Qingdao, Shandong 266071, China \\ Correspondence should be addressed to Ailing Zhang; ailing.zhang@cumt.edu.cn
}

Received 23 November 2021; Revised 17 December 2021; Accepted 21 December 2021; Published 12 January 2022

Academic Editor: Gengxin Sun

Copyright (C) 2022 Ailing Zhang et al. This is an open access article distributed under the Creative Commons Attribution License, which permits unrestricted use, distribution, and reproduction in any medium, provided the original work is properly cited.

\begin{abstract}
With the upgrading of logistics demand and the innovation of modern information technology, the smart logistics platform integrates advanced concepts, technologies, and management methods, maximizes the integration of logistics resources and circulation channels, and effectively improves the efficiency of logistics transactions, but its energy consumption problem is particularly prominent. The study of intelligent measurement and monitoring of carbon emissions in smart logistics is of great value to reduce energy consumption, reduce carbon emissions in buildings, and improve the environment. In this paper, by comparing and analyzing the accounting standards of carbon emissions and their calculation methods, the carbon emission factor method is selected as the method to study the carbon emissions of the smart logistics process in this paper. The working principle of each key storage technology in the smart logistics process is analyzed to find out the equipment factors affecting the carbon emission of each storage technology in the smart logistics process, and the carbon emission calculation model of each key storage technology is established separately by using the carbon emission factor method. Meanwhile, according to the development history of energy consumption assessment, the assessment process of different stages from logistics storage energy consumption assessment to smart logistics energy consumption assessment is analyzed, and based on this, a carbon emission energy consumption assessment framework based on $5 \mathrm{G}$ shared smart logistics is constructed. This paper applies the supply chain idea to define the smart logistics supply chain, constructs a conceptual model of the smart logistics supply chain considering carbon emissions, and at the same time combines the characteristics of the smart logistics supply chain to analyze the correlation between the carbon emissions of the smart logistics supply chain and the related social, environmental, and economic systems.
\end{abstract}

\section{Introduction}

Smart logistics marked by modern information technology has become an important grasp of the supply-side structural reform of the logistics industry, which can effectively integrate social resources, reduce labor costs, meet the personalized needs of consumers, and realize the wisdom of the logistics industry upgrade; energy is the engine and power of human economic development but also an important support for modern social civilization. Historically, with the development of human society and lifestyle changes, all the energy required by humans is increasing [1]. The integration of intelligent carbon emission measurement and monitoring in the process of $5 \mathrm{G}$ shared smart logistics ensures the high- est utilization of resources, the least impact on the surrounding environment, and the greatest overall benefits in the entire life cycle of smart logistics, including product design, product logistics, component assembly, product use, end of life, recycling, and relogistics.

In this paper, the narrow definition of shared intelligent logistics energy consumption assessment refers to the assessment of the degree of energy consumption by integrating the whole life cycle of shared intelligent logistics of products into the service industry, with the whole life cycle of services as the mainline; its broad definition refers to the assessment of the energy consumption of shared intelligent logistics in each stage of the whole life cycle of services and the waste emissions, time spent, and energy flow involved in the 
dynamic tracking, and the assessment is aimed at high-quality and low-consumption logistics process, energy-saving and emission reduction process, and optimal remaining service life to improve the production efficiency and material utilization [2]. The assessment process of energy consumption such as this lacks research on the comprehensive assessment of energy consumption of logistics services throughout the life cycle with the assistance of IoT, big data, digital twin, and other high technologies, and its assessment methods are typically characterized by singularity assessment, process assessment, and fragmentation assessment. Among them, singularity refers to the singularity of the energy consumption assessment method, and the core of the assessment method is mainly the establishment of mathematical models and simulation and calculation based on energy consumption data, etc.; process refers to the fact that the assessment process has a certain time interval and cannot reflect the instantaneous energy consumption of this logistics service comprehensively; fragmentation refers to the fact that the current energy consumption assessment mainly considers the output energy consumption of machinery in the physical space alone or in the information. The modeling, simulation, and prediction of each phase of logistics are cut off in the information space, and there is a lack of efficient collaboration between the whole life cycle of logistics services and energy consumption assessment in the information space and a lack of interaction and integration of physical space and information space [3]. By increasing the investment in ecological compensation, the whole supply chain is changed. Due to the compensation policy and capital investment, the carbon emission of the production link is reduced through green raw materials, order-based production, and energysaving manufacturing, the carbon emission of the storage link is reduced through green packaging and energy-saving storage and direct sales by manufacturers, and the carbon emission of the recycling wisdom is reduced through the optimization of transportation methods and transportation paths. The total carbon emission of the smart logistics supply chain will be reduced by optimizing the transportation method, optimizing the transportation path, and reducing the recycling of smart materials. By comparing and analyzing the accounting standards of carbon emissions and their calculation methods, the carbon emission factor method is selected as the method to study the carbon emissions of the smart logistics process in this paper. The working principle of each key storage technology in the smart logistics process is analyzed to find out the equipment factors affecting the carbon emission of each storage technology in the smart logistics process, and the carbon emission calculation model of each key storage technology is established separately by using the carbon emission factor method. This paper also analyzes the influencing factors of carbon emissions in the logistics process through the study of carbon emissions of storage technologies in the process of shared smart logistics and provides some basis for the development of energy-saving and emission reduction measures in shared smart logistics.

\section{Related Work}

The smart logistics platform emerges under the two-way drive of the changing demand for logistics services and the update of modern information technology such as the Internet, big data, and cloud computing, which provides intelligent modern logistics services to users by integrating groups related to logistics services such as cargo owners, vehicle owners, and storage enterprises. Kuru and Ansell constructed a basic framework of a big data cloud computing-based smart logistics platform consisting of a supply subsystem, demand subsystem, and supervision subsystem [4]. Piovesan et al. proposed to integrate and fuse modern information technology such as cloud computing with traditional logistics information systems and build a "smart cloud logistics" platform system with intelligent decision-making and automatic distribution to solve the logistics problems of e-commerce [5]. The literature [6] outlines the functional planning, target planning, and system framework design of the intelligent logistics platform and gives specific implementation methods. The literature [7] analyzed the construction needs of logistics platforms under cloud computing and constructed a logistics platform framework and functional components of logistics platforms consisting of a cloud platform layer, cloud application layer, and cloud management layer. Logistics platforms were classified according to national, provincial, municipal, and regional levels, and the operation modes and functions of different types of platforms were analyzed. The literature [8] analyzed the operation mechanism of the smart logistics platform based on the bilateral market theory, detailing the platform's incentive mechanism, pricing mechanism, and other operational mechanisms. Lu et al. [9] proposed a functional structure model of a regional logistics information platform and analyzed the role played by the platform in liaising with the government and enterprises. [10] proposed a conceptual model of a smart logistics distribution platform. Matthew [10] proposed a conceptual model of a smart logistics and distribution platform, analyzed the value creation process of the platform stakeholders, and pointed out that the logistics and distribution platform will become a new solution to the logistics and distribution problem. The literature [11] evaluated the business model of traditional logistics information platforms based on the unconfirmed measure of information entropy and other methods, and the results showed that the traditional logistics information platform business model has a single profit model, insufficient standardization of logistics information, and other problems.

The assessment of energy consumption is a key aspect of green logistics, which requires the integration and fusion of energy consumption data in the virtual and physical space to be considered based on the whole life cycle energy consumption data to provide services and applications for energy consumption assessment. As the traditional method of energy consumption assessment ignores the external environment, equipment performance, processing and transportation, and internal environment of materials in use process at different stages of logistics services, it cuts the virtual space and physical space of the assessment process, making it difficult to interact and connect data and information in real time, resulting in the current energy consumption assessment mainly considering the output energy consumption of machinery in the physical space alone or cutting the 
information space. The modeling, simulation, and prediction of each stage of logistics services are not available, and there is a lack of efficient collaboration between the whole life cycle of logistics services and energy consumption assessment in the information space, as well as a lack of real-time interaction and integration between the physical space and the information space, while the carbon emission measurement and assessment based on shared intelligent logistics have greater advantages. Evans and Horak introduce a new global optimization algorithm and propose a green logistics-themed service portfolio as well as multiple data integration and sharing techniques, which provide the conditions for data integration to form a green BOM [12]. The literature [13] researched and developed a new energy estimation framework for calculating energy consumption in the product life cycle and various production processes, which can be extended to different logistics domains and customized for users. Literature [14] developed a new energy estimation framework for calculating energy consumption in the product life cycle and various logistics processes and proposed a requirement for a computational framework for evaluating energy consumption for full product life cycle integration with CAD/CAM systems. Aamir et al. illustrate how simplified design, modeling, and simulation can support the study of life cycle assessment frameworks by reviewing case studies that provide preliminary estimates for material consumption data [15]. The literature [16], on the other hand, presents a methodology for measuring energy efficiency and ecological footprint metrics for product design, modeling, and estimating the total energy consumption of a product based on its various logistical characteristics. Using a probabilistic approach, the literature [17] proposed an energy consumption method on a sequence of relogistics processes. Anser et al. developed a modular simulation model describing a multivariate production system under heterogeneous energy inputs after considering the interdependencies in the production process [18].

\section{5G Research Hypothesis for Value Creation in Shared Smart Logistics}

3.1. Building $5 G$ Shared Smart Logistics Based on the Ecological Value. The intelligent logistics enterprise is the main body of logistics value creation, which provides logistics services for customers through basic activities to achieve profitability and thus create economic value. The realization of economic value is often accompanied by the destruction of the ecological environment, leading to the reduction of ecological value, which is one of the drawbacks of traditional logistics development. Then, while creating economic value, it is necessary to maintain the ability of the enterprise to create ecological value, make the two develop in coordination, and consider the economic value of forest land resources.

Taking the conflict between value and ecological value as an example, the path to internalizing external economy into the coordinated development of both forest land owners' benefits is elaborated from the perspective of the public easement, and the path to the coordinated development of logistics economic value and ecological value is to build a smart logistics system. From the perspective of packaging, smart logistics recommends the packaging size suitable for goods through intelligent packaging technology, uses environmentally friendly materials and electronic face sheets, and implements the promotion of shared express boxes to reduce the waste of logistics packaging, improve the efficiency of packaging utilization, and realize green packaging to improve the ecological value creation ability of enterprises in the packaging link. From the perspective of transportation and distribution, the route is optimized through advanced logistics technologies such as big data and cloud computing to improve the efficiency of transportation and distribution such as vehicle scheduling and cargo monitoring, increase the actual loading rate of vehicles, reduce cargo and energy consumption, and increase the ecological value of the company's transportation and distribution links, so as to realize green transportation and distribution. From the perspective of warehousing, the intelligent warehousing system optimizes warehousing space and inventory and handles special items appropriately to avoid out-of-home situations. From the perspective of warehousing, a series of automated and mechanized equipment is used to load, unload, and sort goods, improve logistics efficiency, reduce labor costs, enhance the ecological value of the enterprise in the process of loading and unloading, and realize green loading and unloading. From the perspective of distribution processing and information service, we improve the efficiency of distribution processing through process optimization and intelligent management and collect, filter, analyze, and utilize logistics information to improve the overall operational efficiency of logistics and realize green distribution processing and information service to improve the ecological value creation ability of enterprises in distribution processing and information service [19]. The process of ecological value creation of intelligent logistics enterprises is shown in Figure 1.

The increase in economic value is the basic goal pursued by every enterprise, and the only way for logistics enterprises to achieve the increase in economic value is to improve their service quality, to improve customer satisfaction; in addition, the ability to save resources, on the one hand, can reduce the operating costs of enterprises and, on the other hand, can reflect the social responsibility of logistics enterprises and establish a good corporate image. The establishment of the corporate image can lay the foundation for long-term development in the future and can continue to bring economic value added to the enterprise. The formation of cost control advantages for the entire logistics industry has epoch-making significance, and intelligent logistics enterprises through the wisdom of technology achieve the optimization of the logistics business process, reduce the work pressure of managers at the same time, and reduce the management costs. Strengthen the effective docking between government departments and between government and enterprises to improve the efficiency of policy implementation and implementation; the level of specialized organization scale in the hub city has a low significance on the impact of comprehensive logistics service capacity in the hub city. However, with the expansion of the logistics demand market, the cost of energy consumption of logistics 


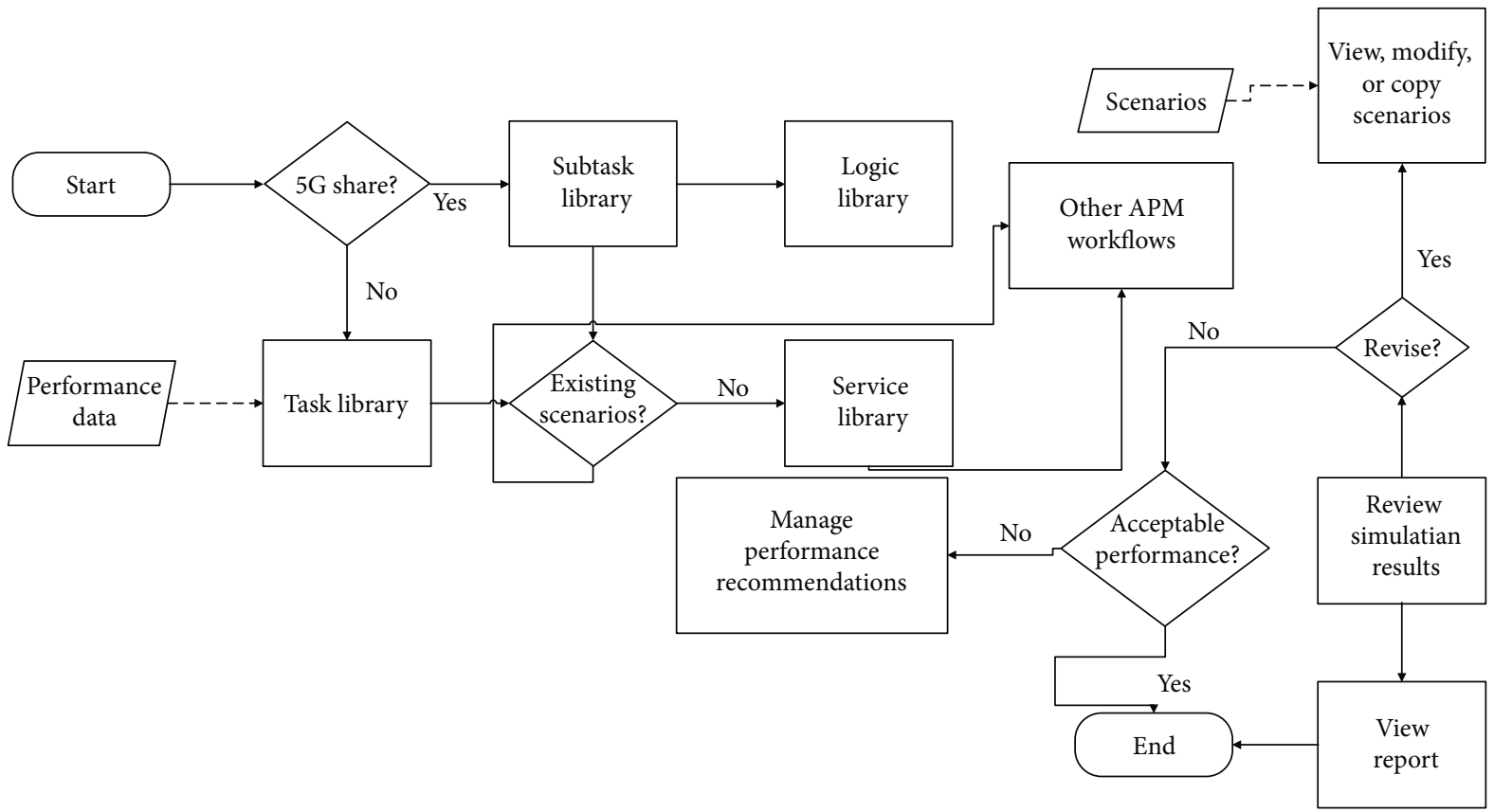

FIGURE 1: 5G shared intelligent logistics ecovalue creation.

enterprises is also rising year by year. The cost control advantages pursued by the intelligent logistics enterprises do not only stop at the reduction of management costs but also stop at the consumption of source costs. In addition, the reduction of the cost of intelligent logistics enterprises to save more liquidity for the development of the enterprise itself, which is conducive to intelligent logistics enterprises to invest more funds in the research and development and application of technology, and the development and successful application of new technologies will further reduce the cost of enterprises and promote the formation of cost control advantages, thus constituting a virtuous circle.

Smart logistics enterprises occupy a place in the market competition under cost control advantages and innovative technology research and development to achieve more value creation; the reduction of enterprise costs brings more intuitive feelings to customers - the reduction of perceived costs and the increase in perceived value, which in turn improves customer satisfaction with smart logistics enterprises [20]. Both the enterprise itself and the customer, through the construction of the cost control advantage, thus realize the value creation of both sides. The cost control advantage can promote the enterprise value creation ability through the indirect role, while the information platform advantage can pull the enterprise value creation through the direct role; secondly, the low-value creation ability is closely related to the business ability; the stronger the business ability, the stronger the enterprise value creation ability, so the business ability advantage is the key to support the enterprise value creation; finally, the operating environment advantage not only promotes the formation of other advantages but also provides a guarantee for the improvement of the overall value creation ability of the enterprise. Through the cultivation of cost control advantage, information platform advantage, busi- ness capability advantage, and operation environment advantage, the value addition of enterprise ecological value, brand value, customer value, and economic value will be gradually realized. The construction of an information platform is the key to building the competitive advantage of intelligent logistics enterprises, since both the enterprise and other stakeholders in the supply chain rely on the convenience brought to them by the platform [21]. First of all, the information platform crosses the spatial obstacle; the original idle logistics resources are integrated and used to improve the overall supply chain and service efficiency, which includes logistics facilities and equipment, logistics network and the community, schools, convenience stores, and other social resources together. Secondly, information sharing becomes the most important function of the information platform. Through the information platform, all participants in the supply chain cooperate and transfer information among enterprises, which alleviates the original "information island" phenomenon among enterprises and helps to improve the overall efficiency of the supply chain and the efficiency of enterprise services, thus realizing value addition. In addition, customers can also experience better logistics services with the help of the information platform, such as real-time understanding of the status of cargo transportation, real-time updating of transportation information, and door-to-door pickup of goods, to meet the diversified needs of customers and ultimately achieve the goal of customer satisfaction, improve the competitiveness of enterprises in the market, and promote value addition.

3.2. Regression Model Construction for Shared Smart Logistics. The fuzzy Borda method adopted in this paper is a method of evaluating single evaluation methods after combining the final scores and rankings derived from each evaluation method based on multiple single evaluation models. 
In this paper, entropy and gray correlation methods are mainly selected to evaluate and calculate six smart logistics enterprises, respectively, and finally, the fuzzy Borda method is used to combine the entropy and gray correlation methods to combine the two evaluation results and finally combine them into a new evaluation model. Virtual space energy consumption-related data specifically includes logistics unit energy consumption simulation data, product logistics energy consumption prediction data, enterprise energy consumption prediction data, logistics energy consumption prediction data, product life cycle energy consumption data, etc.

The concept of entropy is derived from thermodynamics, and it is a measure of the degree of disorder in a system [22]. It can be used to determine the degree of dispersion of an indicator by calculating the magnitude of the entropy value. The smaller the entropy value is, the greater its dispersion degree is and the greater the weight of the indicator in the comprehensive evaluation. The specific steps are as follows.

(1) Construction of the data matrix

Define the matrix as

$$
\left(\begin{array}{ccc}
a_{11} & \cdots & a_{1 n} \\
\vdots & \ddots & \vdots \\
a_{m 1} & \cdots & a_{m n}
\end{array}\right)
$$

$X_{i j}$ is the value of the $j$ th indicator of the $i$ th evaluation object, where $i \in(1, \cdots, n), j \in(1, \cdots, m)$

(2) Calculate the weight of each evaluation object in indicator $j$

$$
K_{i j}=\frac{\partial^{2} \Omega / \partial \mathrm{x} \partial y}{\sum_{i=1}^{n} \sum_{j=1}^{m} x_{i} y_{j}}+C
$$

where $K_{i j}$ is the weight of the $j$ th indicator, $C$ is the correction term, and $\Omega$ is the rate of change of the total indicator.

(3) Calculation of entropy values for each indicator

$$
\eta=-\mu \sum_{i=1}^{n} K_{i j} \Omega_{i j}-\kappa\left|M_{i j}\right|
$$

where $\eta$ is the entropy value and $M_{i j}$ is the coefficient matrix.

(4) Calculation of indicator weights

$$
\varphi_{j}=\frac{L_{j} \cdot \sum_{i=1}^{n} x_{i j}}{\sum_{j=1}^{m} L_{j}}
$$

where $\varphi_{j}$ is the indicator weight and $L_{j}$ is the information redundancy.
(5) Calculate the composite evaluation score for each evaluation subject

$$
g^{n}(x)=\lim _{a \longrightarrow 0} \frac{1}{a^{n}} \sum \varphi_{j}\left(L_{j}-a K_{i j}\right)\left(\begin{array}{l}
n \\
k
\end{array}\right)(-1)^{k}+C,
$$

where $M(x)$ is the electrical resistance of the fiber itself, $p(x)$ is the electrical conductivity, $\varphi$ is the longitudinal length of the fiber, and $A$ is the cross-sectional area of the fiber. The cross-sectional area $A$ of the prepared rGO fibers varies very little during the tensile deformation process and has relatively little effect on the resistance value. The electrical conductivity $p(x)$ will vary somewhat depending on the rGO coating effect, and the preparation process will be influenced by the ratio of GO solution and the number of times the fibers are impregnated in the GO solution. rGO fibers will remain stable in electrical conductivity after forming and can be considered constant. Thus, the magnitude of the resistance of the ego fiber itself is mainly influenced by the longitudinal length $\varphi$, and within a certain range, the resistance value will increase regularly with the production when the fiber is stretched. The above equation only roughly describes some of the factors that cause changes in the resistance of rGO fibers; when the stretching range is too large, the change in resistance does not necessarily show a perfectly linear pattern.

The capacitance is given by the following expression, where $C_{\gamma}$ is the capacitance in the ion equivalent circuit, $C_{\mu}$ is the capacitance per unit area, and $A$ is the area directly opposite to the ends of the capacitor.

$$
C_{\gamma}=C_{\mu} \cdot \int A \cdot M(x) d x .
$$

Here, the capacitance of the semiconductor layer at $z$ position in the direction and width $d z$ is defined, and the expression is

$$
C_{\gamma}(z)=C_{\mu} \cdot \int A \cdot M(x) M(z) d x d z+C_{x 0} .
$$

After applying a positive gate voltage to an organic electrochemical transistor, a dedoping mechanism is used to describe the carrier concentration in the semiconductor layer: cations from the electrolyte are injected into the semiconductor film (here the cations are the charges on this side of the organic semiconductor layer for the equivalent capacitance in the ionic circuit), and each cation injected into the semiconductor layer fills a hole, which is the hole density $K_{\rho}$. According to this mechanism, the expression for the hole density $K_{\rho}$ in the organic semiconductor layer is given by

$$
K_{\rho}(x, y)=\varphi \sum_{y \in \gamma x \in \chi}[p(x, y) \cdot \ln p(x, y)+A x+C y]+\lambda .
$$

Therefore, the doping state is different at each part of the semiconductor layer in the direction $x$, so here the hole 
density at each part of the $K_{\rho a l l}$ semiconductor layer is defined as a function of $x$ :

$$
K_{\rho a l l}=\frac{K_{\rho}(x, y)}{M(x)}=\frac{\varphi \sum_{y \in \gamma} \sum_{x \in \chi}[p(x, y) \cdot \ln p(x, y)+A x+C y]+\lambda}{\varphi \sum_{x \in \chi}\left[p^{2}(x) \cdot \ln p(x)\right]+A x} .
$$

The distribution capacity of the hub city has no significant impact on the scale level of the city's specialized organization, the current logistics network is highly scattered ("fishing net" distribution), various modes of transport coordination are not enough, logistics hubs in promoting industrial division of labor and supply chain synergy integration are not enough, and it is difficult to play the logistics hub agglomeration advantage. Logistics policy has no significant impact on the scale of the city's specialized organization; on the one hand, the number of relevant policies is insufficient, for the scale of the organization, agglomeration development, logistics service capacity, and other policies cannot meet the current requirements of the development of the hub city; on the other hand, the policy is not strong enough; strengthen the effective interface between government departments and government and enterprises, and improve the policy implementation and efficiency. On the one hand, the scale level of logistics organizations in hub cities is not highly specialized; on the other hand, the scale level of logistics organizations in hub cities has not formed an efficient docking channel with logistics service capacity, resulting in a low degree of positive influence.

As shown in Figure 2, the cluster supply chain is a product of the combination of industrial cluster theory and supply chain management, characterized by locality and output, and is a supply chain operation mechanism that expands horizontally based on a single type of supply chain. New business models and service modes in the logistics industry gradually emerge, as well as financing difficulties, excessive transport loads, backward infrastructure construction, and other issues. Cluster supply chain collaboration focuses not only on business cooperation between upstream and downstream enterprises in a single supply chain but also on information resource collaboration between different enterprises in different types of supply chains, so it has the characteristics of both horizontal and vertical integration of supply chains.

The core enterprises are the basis for the formation of individual supply chains, while the synergy between a huge amount of individual supply chains constitutes a more complex supply chain synergy network. Under its leading position in the industry, the core enterprise attracts upstream and downstream enterprises with certain capabilities in adjacent geographic areas to form a vertical supply chain structure. Its strong appeal may come from its strong capital reserve, strong business strength, outstanding resource integration ability, etc. This results in a certain degree of competition among core enterprises in different types of supply chains in a certain region, which negatively affects the effective synergy of clustered supply chains.

\section{Intelligent Carbon Emission Measurement and Monitoring for 5G Shared Smart Logistics}

The carbon emission assessment framework for 5G shared smart logistics is shown in Figure 3, including the physical entity layer and perception layer in physical space and database layer, assessment layer, and optimization layer in virtual space. Among them, the physical entity layer is the level of feedback and corresponds to the virtual model; the perception layer is used to collect and perceive the service process and energy consumption data of logistics services; the database layer is a collection of databases used to hold multisource heterogeneous data; the evaluation layer includes the process of classification, cleaning, processing, and storage of perceived data, as well as the transformation of ECBOM and the evaluation of energy consumption. The optimization layer effectively realizes the optimization of the carbon emission assessment process and results and gives feedback to the physical devices in physical space in real time.

Traditional extraction of elements of physical space entities focuses on high fidelity with virtual models in a static state in an ideal environment, i.e., under conditions of ambient temperature, humidity, air circulation, rapid human reflection, constant rotational speed at rated power, and normal machine operation. However, real physical space entities are subject to large external influences, making it difficult to ensure the accuracy of prediction results and actual output. With the application of $5 \mathrm{G}$ smart sharing technology to the product life cycle, the physical space entity attributes and energy consumption will be mapped into the virtual space with high fidelity and complexity and in real time, thus greatly improving the accuracy of carbon emission assessment.

The sensing layer of carbon emission assessment for 5G shared smart logistics is the collection and acquisition of multisource heterogeneous service process data and energy consumption data based on sensing devices, communication networks, IoT, and manual inputs. Different types of data are acquired in different ways, which can be divided into the perception of fixed data, perception of dynamic data, and complex perception. Among them, the perception of fixed data refers to the acquisition of data such as horizontal and vertical location information in physical space, national standard data, etc., which are fixed for a long time during logistics services, often through scene recognition, SLAM (simultaneous localization and mapping) perception and localization, intelligent collection, manual input, etc. Sensing of dynamic data refers to the flowing data that changes over time throughout the life cycle of logistics services and active monitoring and tracking of data through collaborative control of multiple devices based on the Internet of things, for example, energy consumption data, waste emission data of machinery and equipment, wear and tear of tools, and changes in temperature; in addition to this complex sensing aimed at providing more intelligent, swift, and high-quality logistics services, most of them use expert system energy consumption assessment data fused with traditional mathematical model 


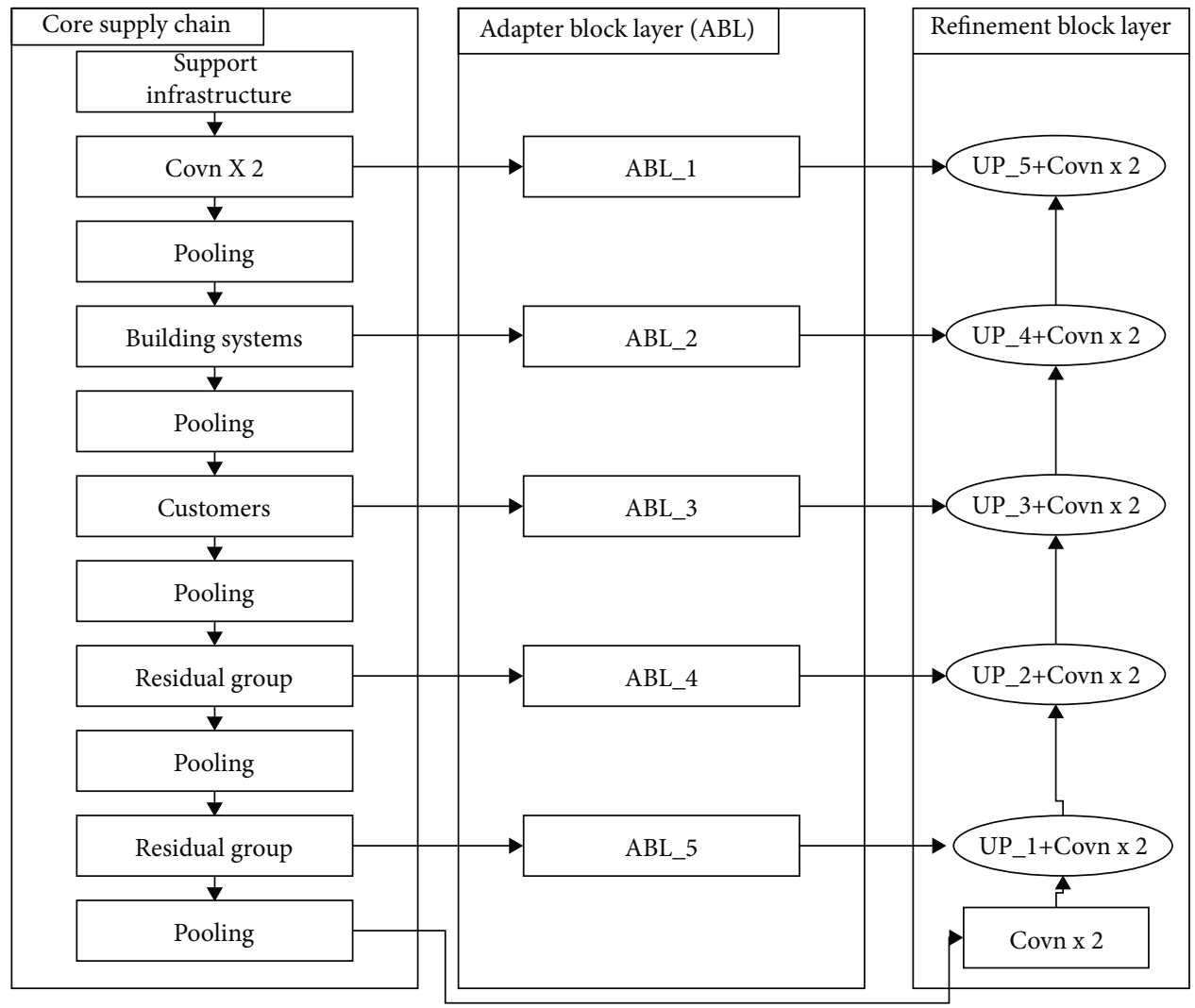

FIGURE 2: Clustered supply chain collaboration.

data to obtain more accurate and reasonable data. The effective sensing and integration of massive heterogeneous, multidimensional, and multisource data provide data source access methods for the application of digital twin technology, real-time mapping, and carbon emission assessment.

The database layer is the database support for carbon emission assessment for $5 \mathrm{G}$ shared smart logistics, which is the process of storing energy consumption-related data involved in the life cycle of logistics services on the cloud platform and is also a collection of all-sided, multiheterogeneous, and complex databases. This database layer is roughly divided into five parts: the collection of physical space data, the collection of virtual space data, the collection of service system data, the collection of fusion data, and the collection of other data. The collection of physical space data is derived from the collection and storage of real-time data in physical space, ephemeral data calls, subjective fuzzy data, etc.; the collection of virtual space data is a collection of models with high-fidelity mapping to physical entities based on the behavior, geometry, rules, etc. of physical space elements; the collection of service system data is a collection of data for energy consumption analysis, energy consumption assessment, and data management within the service system; the collection of fusion data is a collection of data for energy consumption analysis, energy consumption assessment, and data management within the service system. The traditional methods of energy consumption assessment ignore the external environment, equipment performance, processing and transportation, and internal environment of materials in the use process at different stages of logistics services, which cut the virtual space and physical space of the assessment process, making it difficult to interact and connect data and information in real time, resulting in the current energy consumption assessment mainly considering the output energy consumption of machinery in the physical space alone or cutting off in the information space. Modeling, simulation, and prediction of each phase of logistics services lack efficient coordination of the whole life cycle of logistics services and energy consumption assessment in the information space. The collection of fusion data is a collection of intelligent, multifaceted, and real-time evaluation processes based on objective energy consumption, which requires certain learning and self-decision capability to complete high-quality and low-consumption product logistics processes under certain energy consumption standards. This kind of fusion data is also stored in a distributed manner as part of the twin data; the collection of other data is the intellectualized expression of connection rules, interface types, feedback mechanisms, network protocols, optimization algorithms, etc. between different levels. Physical space energy consumption-related data specifically includes logistics unit energy consumption data, logistics service energy consumption data, interenterprise logistics data, product life cycle energy consumption real-time data, material attribute data, water, electricity, and gas and other real-time consumption data, physical space data, human resource data, storage space data, and time-scale data; virtual space energy consumption-related data specifically includes logistics unit 


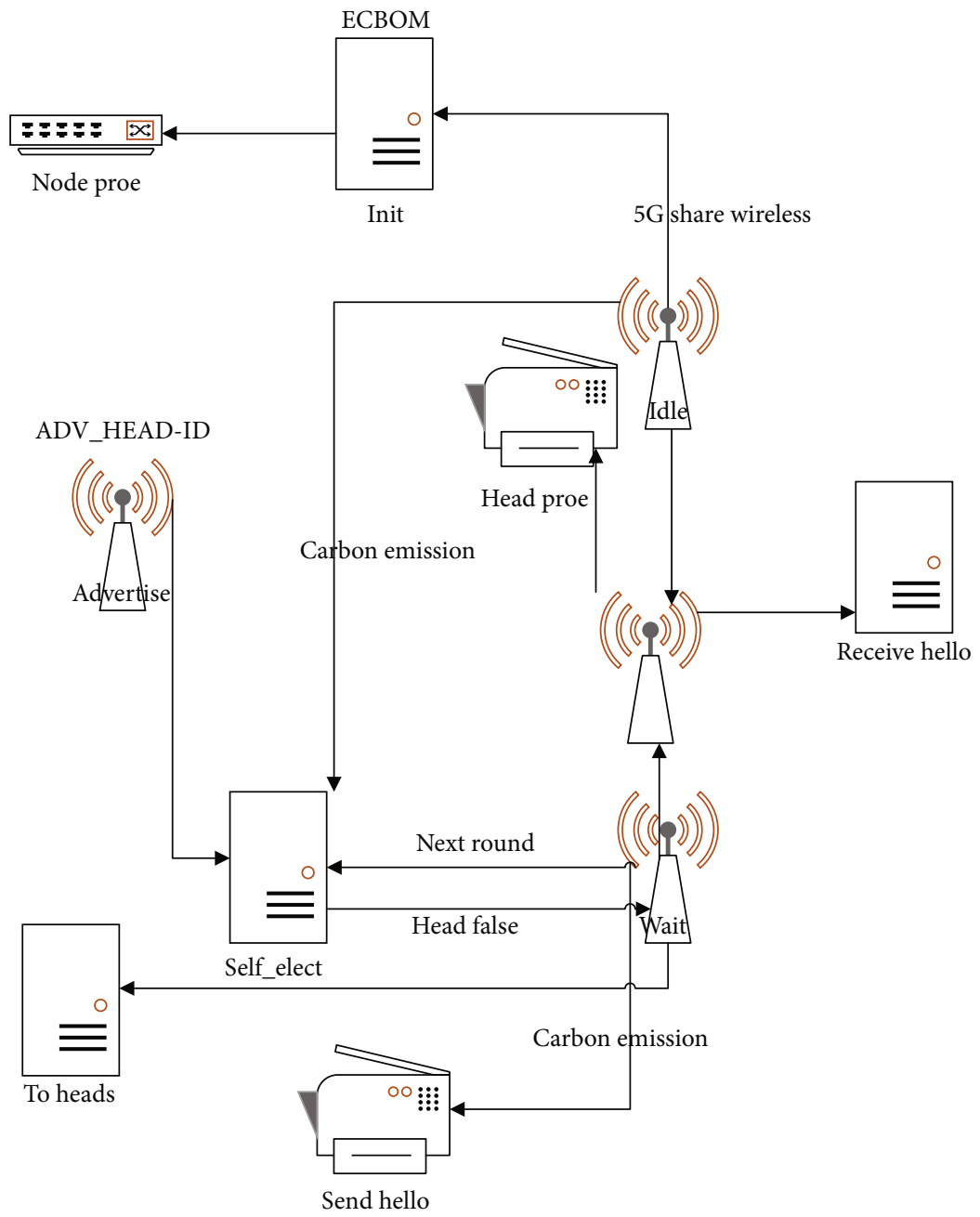

Figure 3: Carbon emission assessment framework for 5G shared smart logistics.

energy consumption simulation data, product logistics energy consumption prediction data, enterprise energy consumption prediction data, logistics energy consumption prediction data, product life cycle energy consumption data, etc.; service system-related data includes real-time energy consumption monitoring service data, energy consumption management service data, product life cycle green design service data, etc.; fusion data includes historical energy consumption optimization data, energy consumption model association fusion data, etc.; other data includes energy consumption source model data, intelligent optimization algorithm data, energy consumption evaluation rule data, etc.

\section{Experimental Verification and Conclusion}

The system dynamic simulation model of the carbon emission system of the smart logistics supply chain can simulate and predict the operation of the system under different policies and contexts and provide a decision basis and data reference for the low-carbon development of the smart logistics supply chain. By comparing the simulation results of carbon emissions and analyzing the extent of the resulting system changes, the best strategy to reduce carbon emissions of the smart logistics supply chain system can be explored. The relevant experimental analysis in this section will define the current state of carbon emissions of the smart logistics supply chain before the change of influencing factors as the basic model, which will be used as the reference value for comparing the simulation results. Combined with the actual situation of the smart logistics supply chain and the related research status, the simulation analysis of the change of the carbon emission factors of the smart logistics supply chain will be carried out in four aspects: increasing the ecological compensation and treatment investment, reducing the unit energy consumption, reducing the carbon emission of the packaging link, and transforming the transportation structure.

The system dynamic simulation model of the carbon emission system of the smart logistics supply chain can simulate different policies and situations to provide a basis for decision-making to reduce carbon emissions in the smart logistics supply chain. The analysis of the changes of system dynamic elements can not only give the development trend under different situations but also clearly understand the specific amount of changes, which is one of the important advantages of system dynamics for simulation. From Figure 4, it can be seen that the production link, the storage 


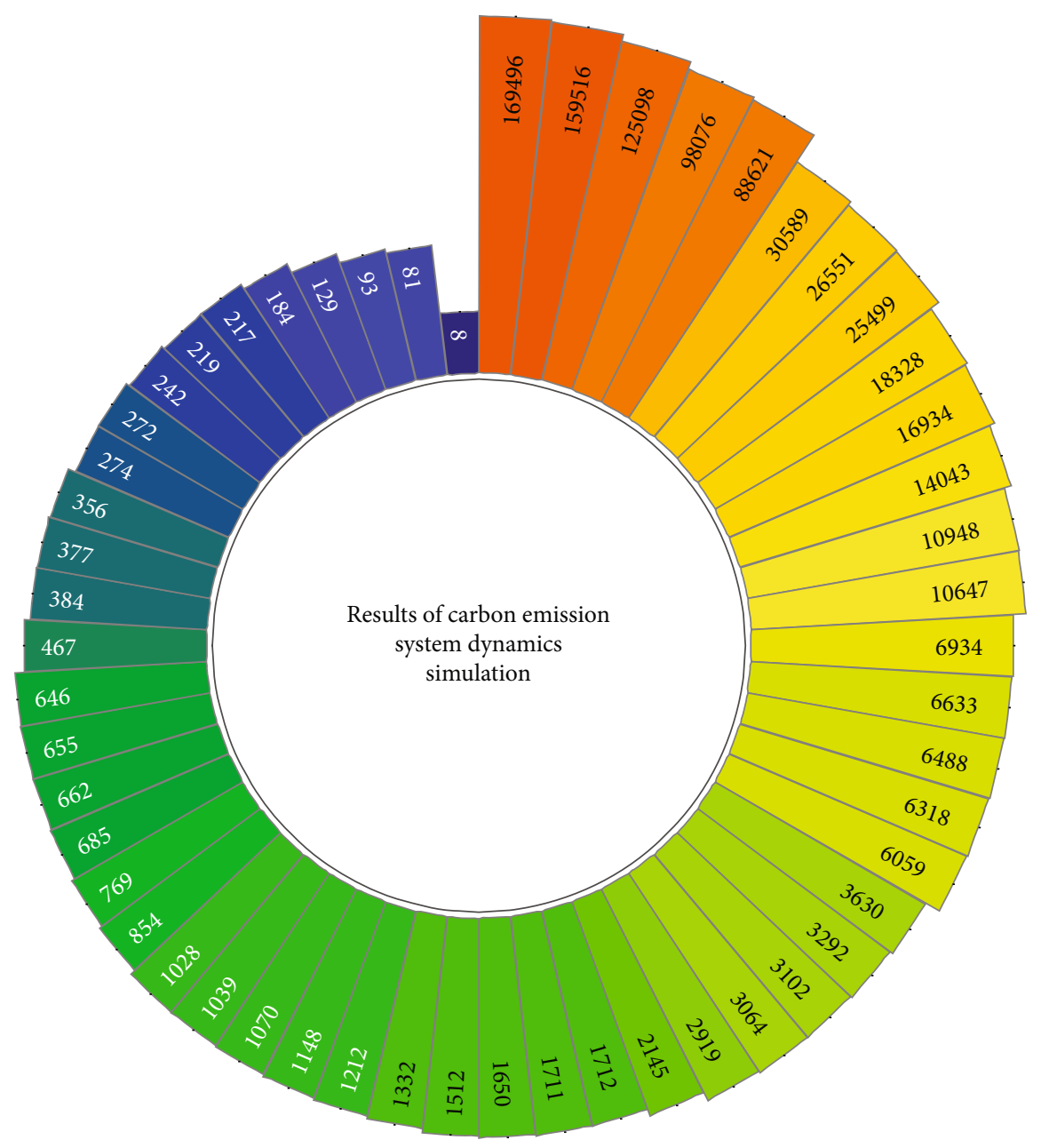

FIGURE 4: Results of carbon emission system dynamic simulation.

link, and the transportation link are the key links that generate more carbon emissions, so the change analysis of the main adjustable factors that generate carbon emissions will be carried out from these three links to simulate the change analysis of carbon emissions. Based on the actual results of the research and the main problems of this supply chain, the detailed question will analyze the change of factors in four aspects, such as the change of energy in the production chain, the change of storage, the change of transforming transportation structure, and the change of increasing ecological compensation investment. As shown in Figure 4, the changes of each influential element of the logistics chain, such as the use of energy-saving logistics equipment and electrical appliances, while increasing the use rate of clean energy, the use of environmentally friendly raw materials, and the use of environmentally friendly simple design to achieve the effect of improving the utilization rate of marginal materials, improve the production capacity to ultimately reduce the overall carbon emissions or to reduce the carbon emissions per unit of intelligent logistics product.

To achieve the overall goal of energy saving and emission reduction, we can start from the perspective of increasing investment in volatile gas and carbon dioxide control and ecological compensation, through increasing investment in ecological compensation, so those producers can consciously reduce their internal carbon emissions, balance the increased costs within the industry due to the increased use of green equipment and green materials, or give some material or policy compensation to those who participate in energy saving and emission reduction in the smart logistics supply chain. By investing ecological compensation funds, the government will promote institutional and energy reforms, such as the adoption of green raw materials and energy-efficient equipment; order-based production, saving production materials and reducing production waste and inventory redundancy, thus increasing the turnover rate of storage capacity; and transportation, through the vehicle management system to optimize transport routes and distribution vehicles to run more efficiently, reducing the carbon emissions of this link. In the long run, sales of products with low-carbon emissions will increase, and government subsidies will allow companies to produce low-carbon and green smart logistics without cost concerns, which will encourage people to start consuming more and more low-carbon products. Increase ecological compensation investment which is from the side or fundamental to change the carbon emissions of the smart logistics supply chain; from Figure 5, it can be seen that, through the government's ecological 

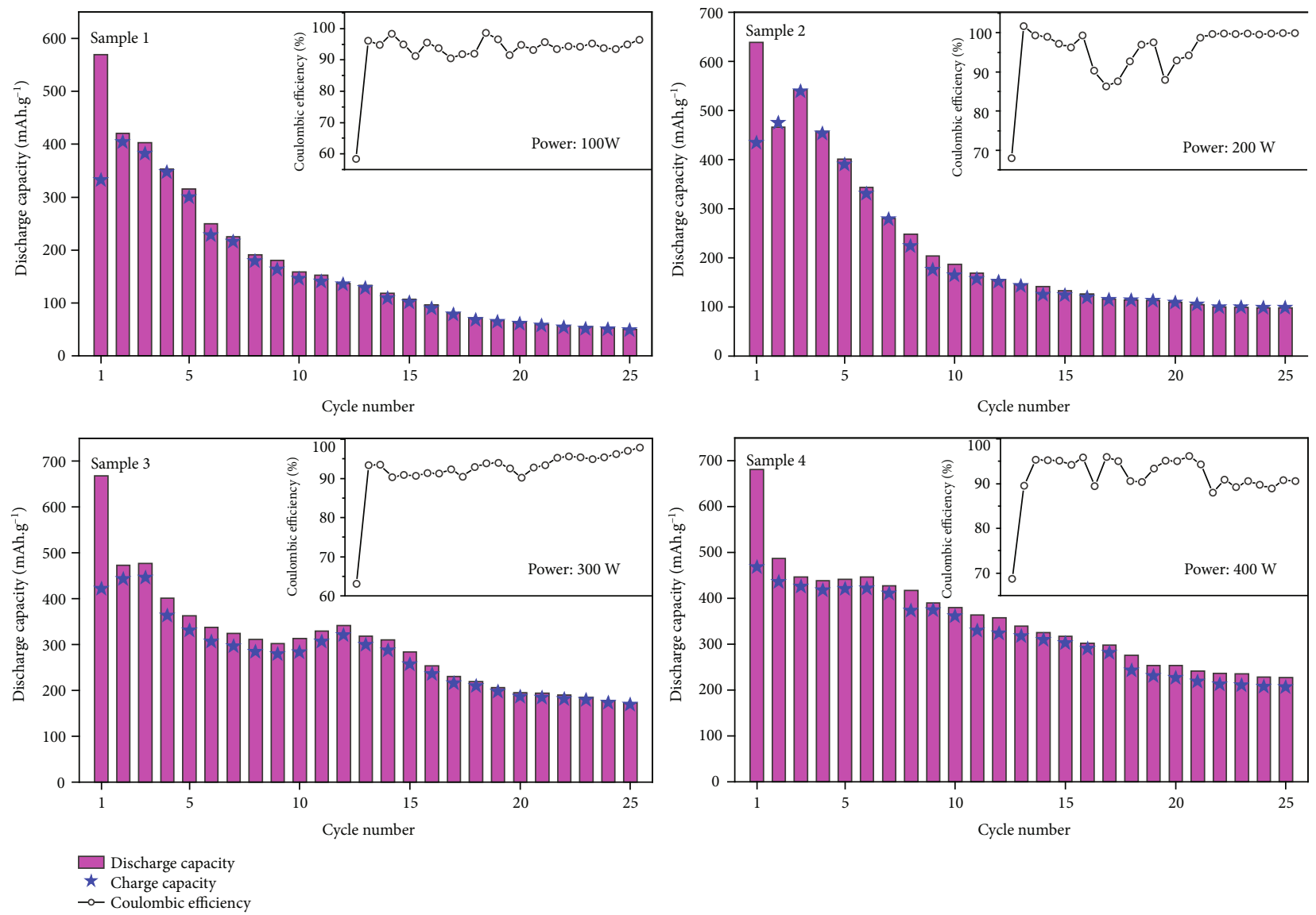

FIgURe 5: Analysis of the impact of changes in ecological offset investments on carbon emissions.

compensation investment, enterprises will have more part of the capital investment, which is invested in production or procurement or transport links or storage links, which can bring a great effect of emission reduction. By increasing ecological compensation investment, the whole supply chain is changed, due to the compensation policy and capital investment, the carbon emission of the production link is reduced through green raw materials, order-based production, and energy-saving manufacturing, the carbon emission of the storage link is reduced through green packaging and energy-saving storage and direct sales by manufacturers, and the carbon emission of the transportation link is reduced by optimizing transportation methods, optimizing transportation routes, and reducing the recycling of the smart materials to reduce the carbon emission of the transportation link; the total carbon emission of the smart logistics supply chain will be reduced.

The transport sector is a key sector, so it is important to control carbon emissions from transport. Figure 6 shows the analysis of carbon emissions from transport, in which the difference in carbon emissions between different modes of transport can be analyzed, and the least carbon-emitting mode of transport can be used and the price of energy can be increased through a carbon tax to reduce carbon emissions from transport. One of the main focuses of the transport chain should be on optimizing the transport structure. There are many irrational transportation processes caused by the irrational process of the smart logistics supply chain, which increases the carbon emission of the smart logistics supply chain. Reduce the intermediate links and ineffective transportation, and adjust the unreasonable process, to realize the low carbonization of the transportation link of the smart logistics supply chain; promoting the use of clean energy is an important strategy to realize the low carbonization of the transportation link. Clustered supply chain collaboration focuses not only on business cooperation between upstream and downstream enterprises in a single supply chain but also on information resource collaboration between different enterprises in different types of supply chains, so it has the characteristics of both horizontal and vertical integration of supply chains. The smart logistics supply chain involves many large and small transportation processes, and the focus of the transportation link should also be on improving transportation services and optimizing distribution routes. Incomplete transportation services and low quality are a major problem in the development of the intelligent logistics industry. The application of intelligent logistics transportation outsourcing should be promoted, regardless of the distance, it will be handed over to professional logistics companies for transportation, and vehicle scheduling will be carried out by professional logistics companies and professional vehicle information management systems. This can be scientific and reasonable, reduce waste in the transportation process, and achieve carbon reduction. The effect of reducing emissions to promote the use of 


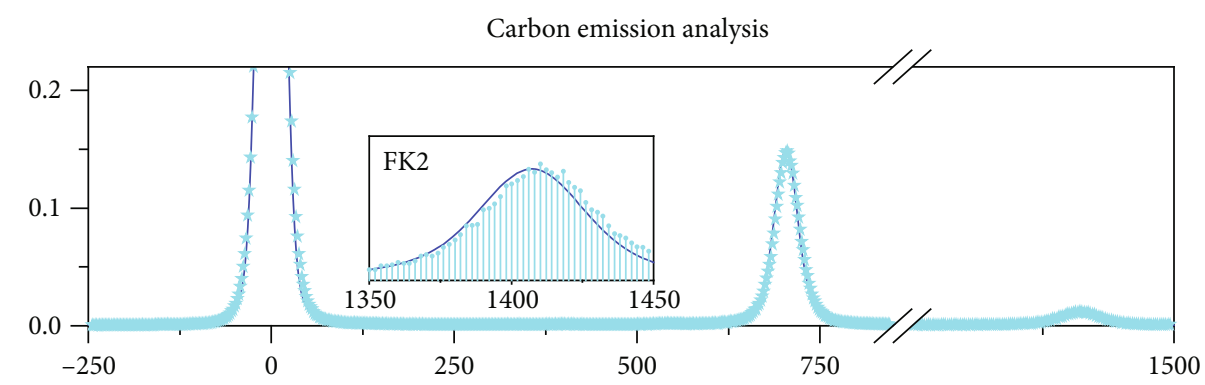

(a)

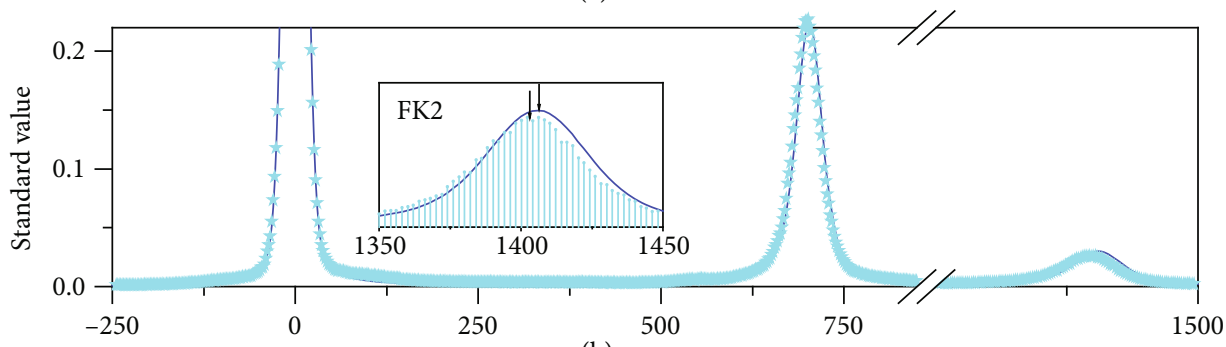

(b)

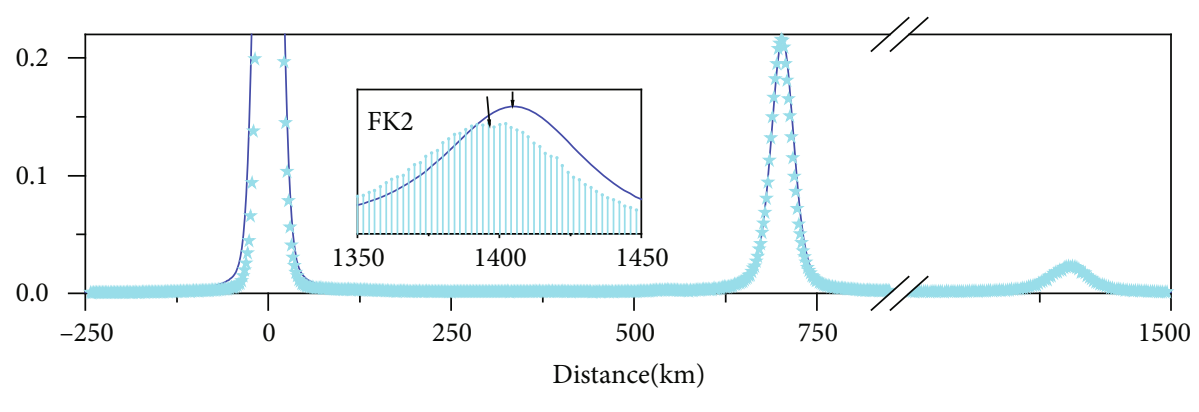

- Fitted

Measured (c)

FIGURE 6: Carbon emission analysis of the transport chain.

clean energy is also an important choice for the low carbonization of the intelligent logistics supply chain.

Transportation, storage, and production link energy and other factors together change, these three links are more carbon emission links, and transportation link adjustment elements are the mode of transport and products with the transport put to improve the full rate, which is the way of change in transport using the same way as the previous section, for storage factors set to the impact of multiple elements: the number of cycles of packaging materials, storage space, lighting equipment, and the results of changes in a variety of elements. Similar to the results of changes in increasing ecological compensation investment, because they are the process of optimizing the overall various key links, with the cost of inputs being different, there will still be some differences in the results; the comprehensive change impact analysis is shown in Figure 7. The influence factors of the three high carbon emission rings, namely, production, warehousing and packaging, and transportation, are integrated, and the change is made comprehensive, and the impact of the change is greatly increased, which can reduce the carbon emission of the three links at the same time and optimize the whole smart logistics supply chain and indeed achieve the effect of reducing the overall carbon emission. The carbon emissions of the production and transportation of the smart logistics supply chain account for $48 \%$ and $34 \%$ of the carbon emissions of the whole supply chain, respectively, indicating that measuring and controlling the production and transportation of the smart logistics supply chain are the focus of the whole smart logistics supply chain carbon emission measurement, and optimizing the production process is also the key to controlling carbon emissions. Therefore, enterprises should improve energy efficiency, use clean production technology while promoting technological innovation, adopt low energy-consuming production equipment, and implement low-carbon production to create lowcarbon smart logistics.

The control of carbon emission of the smart logistics supply chain should pay attention to the development strategy. First of all, we should achieve precise control in the measurement link, choose suitable carbon emission measurement tools, and determine the scope of reducing different greenhouse gas emissions under different environmental performances; adjust the resource structure, transform the development model of the smart logistics industry, and realize green and low-carbon management of the smart logistics supply chain; accelerate product upgrading, comprehensively promote the variety of smart logistics, and comprehensively 


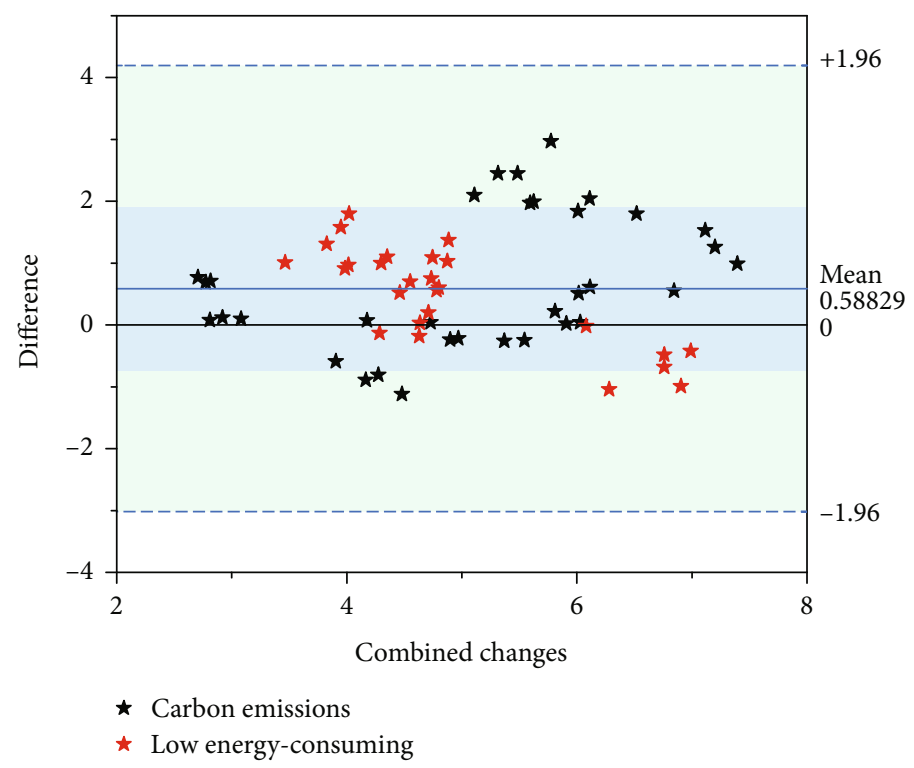

Figure 7: Analysis of the impact of combined changes on carbon emissions.

promote quality and standard improvement; choose lowcarbon emission mechanization equipment to optimize the layout of facilities; and implement government subsidies to regulate the contradiction between the consumer and the supplier through the government subsidy mechanism, to achieve a common fight against carbon emission problems among all parties in the supply chain.

\section{Conclusion}

The logistics industry has entered the track of green development, but it is still in the primary stage. With high pollution and high carbon emission, clean production and low-carbon development are the only ways for the logistics industry, and the study of the carbon emission of the smart logistics supply chain has important theoretical and practical significance for green development and low-carbon economy. To reduce the high-energy consumption and high-carbon emission of the smart logistics industry, the smart logistics supply chain insists on the road of green manufacturing and green production and faces and solves the carbon emission problem of each link together for intelligent development which is the inevitable trend of logistics development. Because intelligence is a typical feature of intelligent logistics, it will continue to improve with the development of artificial intelligence technology, automation technology, and information technology and the degree of intelligence. This is not only limited to the current level. So with the development of the times, intelligent logistics will continue to be given new content.

In this paper, the carbon emission measurement model of the more intelligent logistics supply chain is reasonable and effective through construction. Using the supply chain idea to define the wisdom logistics supply chain, construct a conceptual model of the wisdom logistics supply chain considering carbon emissions, and at the same time combine the characteristics of the wisdom logistics supply chain, analyze the association between the carbon emissions of the wisdom logistics supply chain and the related social, environmental, and economic systems, and conclude that the influencing factors of the wisdom logistics supply chain are mainly energy consumption, production process, process technology, and industrial-scale aspects from the procurement. The model of carbon emission measurement of a smart logistics supply chain is built from five aspects: procurement, production, packaging, storage, and transportation, which enriches the theory of the smart logistics supply chain and provides a reference for carbon emission measurement of smart logistics enterprises. The carbon emission control strategies for each link of the smart logistics supply chain are of practical guidance value. Through the analysis of factor changes, it is proposed that using clean materials and energy-saving equipment instead of the original materials and equipment is an important way to reduce carbon emissions; optimizing the transportation link and using various information systems are important methods to improve the unreasonable reduction of purchasing times and single purchase volume, thus improving the unreasonable transportation situation. Optimization of packaging process and storage function, packaging process design, use of skilled labor, and good storage management are important means to reduce carbon emissions in packaging and storage.

\section{Data Availability}

The data used to support the findings of this study are available from the corresponding author upon request.

\section{Conflicts of Interest}

The authors declare that they have no known competing financial interests or personal relationships that could have appeared to influence the work reported in this paper. 


\section{References}

[1] Y. Ding, M. Jin, S. Li, and D. Feng, "Smart logistics based on the Internet of things technology: an overview," International Journal of Logistics Research and Applications, vol. 24, no. 4, pp. 323-345, 2021.

[2] S. H. Alsamhi, O. Ma, M. S. Ansari, and Q. Meng, "Greening Internet of things for greener and smarter cities: a survey and future prospects," Telecommunication Systems, vol. 72, no. 4, pp. 609-632, 2019.

[3] M. S. Răboacă, N. Bizon, and P. Thounthong, "Intelligent charging station in $5 \mathrm{G}$ environments: challenges and perspectives," International Journal of Energy Research, vol. 45, no. 11, pp. 16418-16435, 2021.

[4] K. Kuru and D. Ansell, "TCitySmartF: a comprehensive systematic framework for transforming cities into smart cities," IEEE Access, vol. 8, pp. 18615-18644, 2020.

[5] N. Piovesan, D. A. Temesgene, M. Miozzo, and P. Dini, "Joint load control and energy sharing for autonomous operation of 5G mobile networks in micro-grids," IEEE Access, vol. 7, pp. 31140-31150, 2019.

[6] K. Muhammad, J. Lloret, and S. W. Baik, "Intelligent and energy-efficient data prioritization in green smart cities: current challenges and future directions," IEEE Communications Magazine, vol. 57, no. 2, pp. 60-65, 2019.

[7] M. A. Habibi, M. Nasimi, B. Han, and H. D. Schotten, "A comprehensive survey of RAN architectures toward 5G mobile communication system," IEEE Access, vol. 7, pp. 70371-70421, 2019.

[8] X. Chen, "The development trend and practical innovation of smart cities under the integration of new technologies," Frontiers of Engineering Management, vol. 6, no. 4, pp. 485-502, 2019.

[9] H. P. Lu, C. S. Chen, and H. Yu, "Technology roadmap for building a smart city: an exploring study on methodology," Future Generation Computer Systems, vol. 97, pp. 727-742, 2019.

[10] E. Mathew, "Swarm intelligence for intelligent transport systems: opportunities and challenges," Swarm Intelligence for Resource Management in Internet of Things, pp. 131-145, 2020.

[11] L. Belli, A. Cilfone, L. Davoli et al., "IoT-enabled smart sustainable cities: challenges and approaches," Smart Cities, vol. 3, no. 3, pp. 1039-1071, 2020.

[12] V. Evans and J. Horak, "Sustainable urban governance networks, data-driven Internet of things systems, and wireless sensor-based applications in smart city logistics," Geopolitics, History, and International Relations, vol. 13, no. 2, pp. 6578, 2021.

[13] M. Autili, L. Chen, C. Englund, C. Pompilio, and M. Tivoli, "Cooperative intelligent transport systems: choreographybased urban traffic coordination," IEEE Transactions on Intelligent Transportation Systems, vol. 22, no. 4, pp. 2088-2099, 2021.

[14] Y. Noh and J. Y. Ro, "A study on the service provision direction of the National Library for Children and Young Adults in the 5G era," International Journal of Knowledge Content Development \& Technology, vol. 11, no. 2, pp. 77-105, 2021.

[15] M. Aamir, S. Masroor, Z. A. Ali, and B. T. Ting, "Sustainable framework for smart transportation system: a case study of Karachi," Wireless Personal Communications, vol. 106, no. 1, pp. 27-40, 2019.
[16] H. Lu, L. Guo, M. Azimi, and K. Huang, "Oil and gas 4.0 era: a systematic review and outlook," Computers in Industry, vol. 111, pp. 68-90, 2019.

[17] R. Philipp, G. Prause, E. O. Olaniyi, and F. Lemke, “Towards green and smart seaports: renewable energy and automation technologies for bulk cargo loading operations," Environmental and Climate Technologies, vol. 25, no. 1, pp. 650-665, 2021.

[18] M. K. Anser, M. Ahmad, M. A. Khan et al., "The role of information and communication technologies in mitigating carbon emissions: evidence from panel quantile regression," Environmental Science and Pollution Research, vol. 28, no. 17, pp. 21065-21084, 2021.

[19] H. Treiblmaier, A. Rejeb, and A. Strebinger, "Blockchain as a driver for smart city development: application fields and a comprehensive research agenda," Smart Cities, vol. 3, no. 3, pp. 853-872, 2020.

[20] M. A. Adedoyin and O. E. Falowo, "Combination of ultradense networks and other $5 \mathrm{G}$ enabling technologies: a survey," IEEE Access, vol. 8, pp. 22893-22932, 2020.

[21] T. Yigitcanlar, N. Kankanamge, and K. Vella, "How are smart city concepts and technologies perceived and utilized? A systematic geo-Twitter analysis of smart cities in Australia," Journal of Urban Technology, vol. 28, no. 1-2, pp. 135-154, 2021.

[22] Z. Tong, F. Ye, M. Yan, H. Liu, and S. Basodi, "A survey on algorithms for intelligent computing and smart city applications," Big Data Mining and Analytics, vol. 4, no. 3, pp. 155-172, 2021. 